\title{
Increased expression of nucleophosmin/B23 in hepatocellular carcinoma and correlation with clinicopathological parameters
}

\author{
J-P Yun ${ }^{*, 1,2,4}$, J Miao $^{3,4}$, GG Chen ${ }^{3}$, Q-H Tian ${ }^{1,2}$, C-Q Zhang', J Xiang ${ }^{1,2}$, J Fu ${ }^{1,2}$ and PBS Lai ${ }^{3}$ \\ 'State Key Laboratory of Oncology in Southern China, Cancer Center, Sun Yat-Sen University, Guangzhou, China; 'Department of Pathology, Cancer \\ Center, Sun Yat-Sen University, Guangzhou, China; ${ }^{3}$ Department of Surgery, The Chinese University of Hong Kong, SAR Hong Kong, China
}

Nucleophosmin (NPM, B23, numatrin, NO38) is an abundant nucleolar phosphoprotein involved in multiple cellular functions. Previous evidence indicates that high-level expression of NPM causes uncontrolled cell growth and suggests that NPM may have oncogenic potential. In this study, we examined NPM expression in 103 paired cases of hepatocellular carcinoma (HCC), I2 cases of hepatic focal nodular hyperplasia, 17 cases of liver tissue adjacent to a hepatic haemangioma, and series of array tissues from normal human organs and malignancies using a monoclonal antibody against NPM and reverse transcription-PCR techniques, Western blot analysis, immunohistochemistry, and immunocytofluorescence. Our data indicated that NPM expression was significantly higher in $\mathrm{HCC}$ than in the non-malignant hepatocytes $(\mathrm{P}<0.00 \mathrm{I})$. Nucleophosmin was weakly expressed in hepatocytes from a 5-month-old embryo and in stationary hepatocytes of healthy adults. Moreover, enhanced expression of NPM in HCC correlated with the level of PCNA $\left(R^{2}=0.5639\right)$ and with the clinical prognostic parameters such as serum alpha fetal protein level, tumour pathological grading, and liver cirrhosis $(P<0.05)$. Our results suggest that NPM may play an important role in the progression of tumorigenesis and that NPM may serve as a potential marker for HCC.

British Journal of Cancer (2007) 96, 477-484. doi:10.1038/sj.bjc.6603574 www.bjcancer.com

Published online 23 January 2007

(c) 2007 Cancer Research UK

Keywords: nucleophosmin; B23; hepatocellular carcinoma; PCNA; cell proliferation; malignancy

Hepatocellular carcinoma (HCC) is one of the most prevalent malignancies worldwide, with an annual incidence of 250000 diagnoses. Although the process of hepatocarcinogenesis has not yet been fully elucidated, it is considered a multifactorial and multistep process that involves the activation of oncogenes or the inactivation of tumour suppressor genes during the different stages of HCC progression. Several studies have recently shown that activation of oncogenes was involved in hepatocarcinogenesis, genes such as cyclin D1 (Deane et al, 2001), the transcription factor Yin Yang 1 (YY1) (Gordon et al, 2006), and the hedgehog pathway (Sicklick et al, 2006). Overexpression of cyclin D1 has been shown to be sufficient for the initiation of hepatocellular carcinogenesis in a transgenic mouse model (Deane et al, 2001). The transcription factor YY1 is considered to possess the potential to act as an initiator of tumorigenesis and may serve as both a diagnostic and prognostic tumour marker (Gordon et al, 2006). Overexpression and tumorigenic activation of the Smoothened (SMO) protooncogene mediates c-myc overexpression, suggesting that SMO may also be a prognostic factor in HCC tumorigenesis (Sicklick et al, 2006).

Nucleophosmin (NPM) is a major nucleolar phosphoprotein implicated in multiple cellular functions, including ribosomal

\footnotetext{
* Correspondence: Dr J-P Yun, Cancer Center, Sun Yat-Sen University, no. 65I, East Dongfeng Road, Guangzhou 510060 China;

E-mail: yunjp@mail.sysu.edu.cn

${ }^{4}$ These authors contributed equally to this work.

Received 4 October 2006; revised 28 November 2006; accepted 28 November 2006; published online 23 January 2007
}

protein assembly and transport (Verheggen et al, 2000; Huang et al, 2005), centrosome duplication (Okuda et al, 2000; Okuda, 2002; Grisendi et al, 2005), molecular chaperone activity in preventing protein aggregation (Hingorani et al, 2000; Szebeni et al, 2003), and regulating the activity of the tumour suppressors p53 (Colombo et al, 2002; Li et al, 2004; Maiguel et al, 2004) and p14 ${ }^{\mathrm{ARF}}$ (Itahana et al, 2003; Bertwistle et al, 2004; Brady et al, 2004). Earlier studies have shown that the level of NPM is markedly and promptly increased in association with cell commitment to mitogenesis (Feuerstein and Mond, 1987; Feuerstein et al, 1988). It has also been shown that NPM is a direct target of the Myc transcription factor during ribosomal biogenesis (Zeller et al, 2001) and cyclin E/CDK2 kinase activity during centrosome duplication (Okuda et al, 2000). Nucleophosmin physically interacts with several other cellular factors such as the retinoblastoma susceptibility gene product pRB (Takemura et al, 1994, 1999), the cell cycle G2-M checkpoint factor Gadd45a (Gao et al, 2005), and the tumour suppressor p14 ${ }^{\mathrm{ARF}}$ (Itahana et al, 2003). Mounting evidence has linked excessive NPM to cellular transformation and ontogenesis (Pulford et al, 2001). Nucleophosmin overexpression is frequently found in human cancers, including cancers of the stomach (Tanaka et al, 1992), colon (Nozawa et al, 1996), bladder (Tsui et al, 2004), prostate (Subong et al, 1999), thyroid (Onda et al, 2004), ovary (Zhang, 2004), and myeloid and lymphoid cells (Schnittger et al, 2005). It has been shown that overexpression of NPM in bladder cancer is independently associated with cancer recurrence and progression to more advanced stages, suggesting that NPM overexpression is an important prognostic indicator for bladder cancer recurrence (Tsui et al, 2004). Furthermore, ectopic overexpression of NPM in 
NIH3T3 cells results in malignant transformation (Kondo et al, 1997). These findings further suggest that NPM is involved in the regulation of cellular growth in various normal and neoplastic cells and may be used as a clinical indicator for cancer patients.

The present study is aimed at investigating the potential roles of NPM in HCC using a systematic analysis on the levels of NPM in a large number of human HCC and non-malignant liver lesions, in normal human adult and embryo tissues, and to address the correlation between NPM expression and the clinical parameters.

\section{MATERIALS AND METHODS}

\section{Nucleophosmin recombinant protein and monoclonal antibody}

Human NPM was PCR amplified from cDNA (catalogue no. MHS1011-58590, clone ID: 2821577, OpenBioSystem, Huntsville, AL, USA) using specific primers according to NPM1 gene source sequence $\left(5^{\prime}\right.$-cgcggatccgaagattcgatggacatggac and $3^{\prime}$-cccaagcttaa gagacttcctccactgcca). The gene was then cloned into the Pqe30 vector (catalogue no. 33203, Qiagen, Valencia, CA, USA) and expressed in an Escherichia coli expression system to obtain recombinant NPM protein. The purified recombinant protein was used to generate a monoclonal antibody using routine hybridoma cell techniques. The titre and specificity of the antibodies were analysed and characterised by Western blot analysis and immunocytofluorescene. A PCNA monoclonal antibody was purchased (Zymed, South San Francisco, CA, USA).

\section{Clinical samples and tissue arrays}

Samples from 132 cases with liver disease and their clinical material were collected from the files of the Cancer Center of Sun Yat-Sen University, Guangzhou, China. These cases included 103 cases of HCC, 12 cases of hepatic focal nodular hyperplasia (FNH), and 17 cases of hepatic haemangiomas. All of the tissue blocks were sectioned for immunohistochemistry of NPM and PCNA. Ten paired cases of HCC tissue along with the adjacent hepatic tissue from the tissue bank department of this cancer centre were collected for reverse transcription-PCR (RT-PCR) and Western blot analyses. Another tissue array with sections from multiple organs, including normal and diseased tissues for immunohistochemistry, was purchased from Cybrdi Biotech Co (catalogue no. CC00-11-002, CC00-11-003, CC00-01-004, EC01-01-006, NC03-01001, Cybridi, Xian City, China). This array included normal adult tissues; normal embryo tissues (5 months); diseased tissues, including malignancies from liver, brain, lung, kidney, stomach, colon, breast, cervix, prostate, and skin; as well as cells from different origins such as epithelia, non-epithelia, and lymphocytes. All of the human specimens in the study were approved by the Independent Ethics Committee of the Cancer Center of Sun Yat-Sen University.

\section{Cell culture, immunocytofluorescence, and Western blot analysis}

Six hepatoma cell lines including Hep G2, Huh-7, PLC/PRF/5, SKHep-1, Chang, and Hep-3B were cultured as reported previously (Yun et al, 2003; Miao et al, 2006). Briefly, for immunocytofluorescence, the cultured cells were fixed for $10 \mathrm{~min}$ and rinsed with PBS. The cells were then incubated with the primary monoclonal antibody (NPM, 1:1000) overnight, followed by incubation with a fluorescence-conjugated secondary antibody for $1 \mathrm{~h}$, and finally dehydrated and mounted. The fluorescent signal was observed under fluorescent microscopy (Yun et al, 2003). For immunolabelling, lysates from the tissue samples were prepared as reported previously (Yun et al, 2003; Miao et al, 2006). One hundred micrograms of each lysate was separated by SDS-PAGE. The proteins were transferred onto blotting membranes. After blocking, the membranes were incubated overnight with mouse monoclonal antibody against NPM, rabbit polyclonal antibody against PCNA (FL-261, Santa Cruz Inc., Santa Cruz, CA, USA), and mouse monoclonal antibody against GAPDH (Kangchen Biotech, Shanghai, China) (NPM, 1:2000; PCNA, 1:1000; GAPDH, 1:1000), followed by incubation with a horseradish peroxidase-conjugated IgG. The blots were then visualised with an ECL kit (Amersham Life Science, Piscataway, NH, USA) and exposed for $30 \mathrm{~s}$ (NPM) and $1 \mathrm{~min}$ (PCNA, GAPDH) to X-ray film. The bands were analysed using the Quantity One ${ }^{\mathrm{TM}}$ Software (Bio-Rad, Hercules, CA, USA).

\section{Reverse transcriptase-PCR}

Total RNA was extracted from 10 paired samples of frozen HCC tissue and adjacent hepatic tissue using the Trizol method (Gibco, Carlsbad, CA, USA) according to the manufacturer's instructions. One microgram of RNA sample was reverse transcribed with oligo(dT)15 primers (Promega, Madison, WI, USA) to obtain single-stranded cDNA. One-tenth of the product was used as template in PCR amplification for 28 cycles in a thermal cycler. Each cycle consisted of $30 \mathrm{~s}$ denaturation at $94.5^{\circ} \mathrm{C}, 30 \mathrm{~s}$ annealing at $55^{\circ} \mathrm{C}$, and $1 \mathrm{~min}$ extension at $72^{\circ} \mathrm{C}$. Under these conditions, the amplifications occurred in a linear exponential phase. The following primers were used: NPM forward, 5'-CAC CCG ATG GAA GAT TC- $3^{\prime}$; NPM reverse, 5'-GGA CAG CCA GAT ATC AAC T-3'; G3PDH forward, 5'-AAA TCC CAT CAC CAT CTT CC-3'; and G3PDH reverse, $5^{\prime}$-TCC ACC ACC CTG TTG CTG TA-3'. The PCR products were analysed by $1.0 \%$ agarose gel electrophoresis. The abundance of PCR signals was determined using the Quantity One $^{\mathrm{TM}}$ Software (Bio-Rad, Hercules, CA, USA).

\section{Immunohistochemistry}

One hundred thirty-two sample blocks were sectioned for immunohistochemistry of NPM and PCNA. The above tissue array sections were prepared for immunohistochemistry of NPM with a three-step immunoperoxidase method using a Strept-Avidin Biotin kit (Dakopatts, Glostrup, Denmark) as previously described (Yun et al, 2003, 2004). Briefly, after blocking, the sections were incubated in primary antibodies overnight (NPM, 1:400; PCNA, $1: 400)$, followed by incubation in secondary antibodies, and further incubation with the Strept-Avidin Biotin complex (Dakopatts, Denmark). Reactivity was developed in chromogen DAB solution. After contrast staining, the sections were dehydrated and mounted. A brown particle in a cell was considered as positive labelling. These sections were observed under light microscopy and the staining intensities were assessed by two pathologists - Dr JP Yun and Dr J Xiang.

\section{Statistical analysis}

After immunohistochemistry of NPM and PCNA of tissue from cases with liver diseases, four types of nuclear staining intensities were discovered. These were classified as strongly positive $(+++)$, intermediately positive $(++)$, weakly positive $(+)$, or negative $(-)$. For each case, 1000 cells in one case were randomly selected and counted, scoring for these four intensities. For factor analysis, the percentages of the four intensities were transformed to one Ridit value for each case that was representative of the NPM or PCNA expression intensity. The usual tests (comparison of means, $t$-test, and one-way ANOVA) were then used to analyse the differences of NPM or PCNA expression among the different groups. The Pearson correlation was employed to examine the relationship between NPM and PCNA expression. Statistical significance was initially set at $P<0.05$. 


\section{RESULTS}

\section{Nucleophosmin expression in the HCC tissues}

In seven out of 10 cases, NPM mRNA expressions were higher in HCC tissues than in the paired liver tissue adjacent to the tumours (samples 2-5 and 7-9), as determined by RT - PCR (Figure 1). In three cases, NPM expressions of the HCC tissues were found to be lower than those in the adjacent healthy tissues (samples 1 and 10)

$M N_{1} T_{1} N_{2} T_{2} N_{3} T_{3} N_{4} T_{4} N_{5} T_{5} N_{6} T_{6} N_{7} T_{7} N_{8} T_{8} N_{9} T_{9} N_{10} T_{10}$

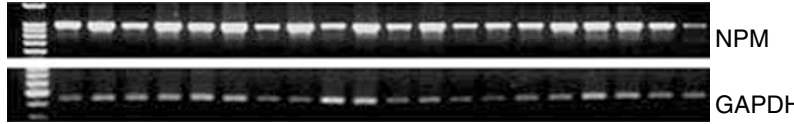

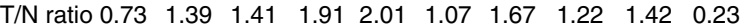

Figure I Nucleophosmin expression was detected in clinical samples with HCC by RT-PCR. Ten paired samples of HCC tissue and the corresponding liver tissue adjacent to the tumour were used. Samples TI 10 are $\mathrm{HCC}$ tissues, whereas $\mathrm{NI}-10$ designate the corresponding adjacent healthy tissue samples. M: marker. Expression of the housekeeping gene, GAPDH, serves as a control.

A
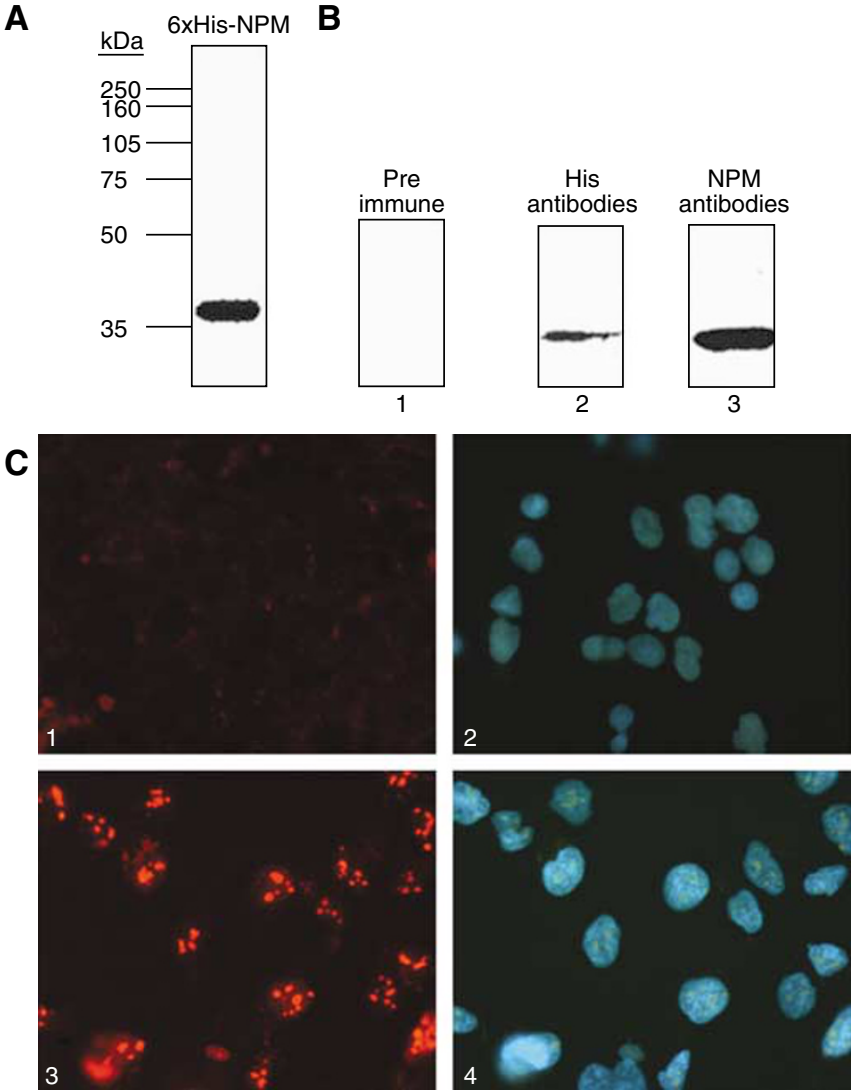

Figure 2 Characterisation of NPM antibodies. (A) The identity of NPM recombinant protein $(\mathrm{I} \mu \mathrm{g})$ was verified by Western blot analysis with an anti-NPM antibody. (B) The immunoreactivity of NPM protein to different antibodies ( $10 \mathrm{ng}$ per lane) was analysed by Western blot using preimmune serum (lane 1), an anti-His antibody (1:4000, lane 2), or anti-NPM antibody ( $1: 2000$, lane 3$)$. The recombinant NPM proteins were detected by His antibody or NPM antibody, but not by the preimmune serum. $(\mathbf{C})$ Immunocytofluorescence analysis using NPM antibodies. Image I: cultured Hep B3 cells were stained with preimmune serum; image 2: the same cells as in image I were stained with DAPI; image 3: cultured Hep B3 cells were stained with anti-NPM antibodies; image 4: the same cells as in image 3 were stained with DAPI. (All fields $\times 400$.) and nearly same as that in the adjacent healthy tissue (sample 6). The expression levels of NPM in these samples with HCC were on average 1.31-fold higher than in the paired healthy tissue. These results indicated that NPM mRNA was expressed at a higher level in HCC than in the hepatocytes adjacent to the tumour.

For use in immunolabelling studies, NPM monoclonal antibodies were raised against recombinant NPM protein (with a molecular weight of approximately $35 \mathrm{kDa}$ ) and characterised by Western blot and immunocytofluorescence (Figure 2). In total, one clone was screened to produce specific monoclonal antibodies against NPM that were used to immunolabel this protein in Western blot analysis, immunofluorescence, and immunohistochemistry.

Nucleophosmin protein expressions in the above 10 paired samples were analysed by Western blot analysis. In seven out of 10 samples, expression of this protein was apparently higher in HCC tissues than in the liver tissue adjacent to the tumour; however, there was no apparent difference of expression in three cases. The mean expression level of the HCC samples was 1.88 -fold higher than the paired liver tissue. The expression pattern of NPM was similar to that of PCNA (Figure 3A). These results indicate that NPM protein is expressed at a higher level in HCC than in hepatocytes adjacent to the tumour.

The subcellular expression of NPM was determined by immunocytofluorescence. It was found to localise predominantly in the nucleoli of six cultured cell lines and weakly in the nuclei of some cells such as Hep G2, PLC/PRF/5, SK-Hep-1, and Hep-3B (Figure $3 \mathrm{~B}$ ). This may suggest that NPM functions primarily in the nuclei of these cultured hepatoma.

\section{Expression of NPM and/or PCNA in HCC and non- malignant liver diseases}

As shown in Figures 4 and 5, NPM was more highly expressed in HCC (group 1), as determined by immunohistochemistry, was mainly located in the nuclei of the cancer cells, and was higher than that in the hepatocytes with non-malignant diseases (groups $2-4)$ in staining intensity or the total positive cell number.

\section{A}

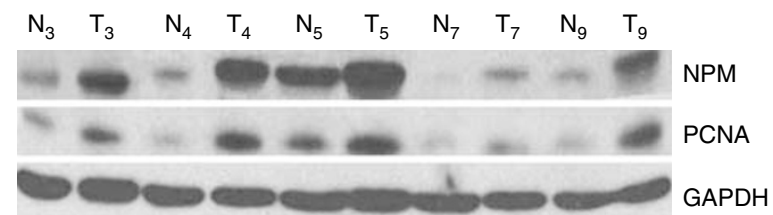

B
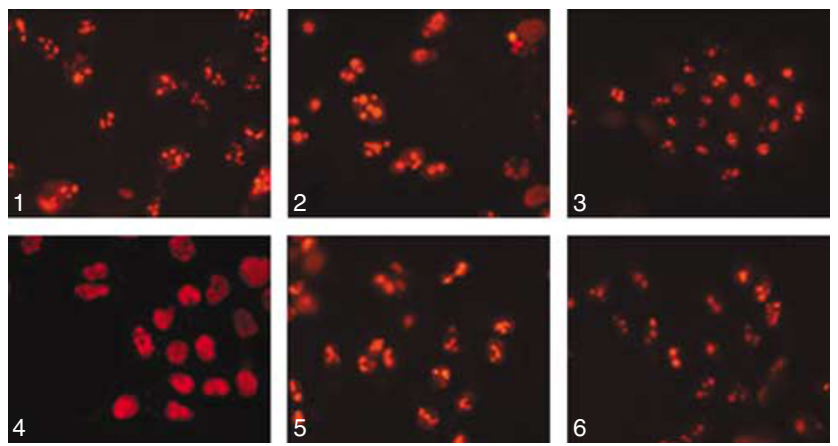

Figure 3 Nucleophosmin protein expression was detected in clinical samples with HCC by Western blot analysis and immunocytofluorescence. (A) Higher expression of NPM was found in HCC tissue samples (T3-5, T7, and T9) than in the corresponding adjacent tissue (N3-5, N7, and N9). This was similar to the PCNA expression trend in the same samples. As a control, GAPDH was used. (B) Nucleophosmin subcellular localisation was also examined in these cells. Hep 3B (image I), PLC/PRF/5 (image 2), Huh7 (image 3), Hep G2 (image 4), SK-Hep-I (image 5), and Chang (image 6). (All fields, $\times 400$.) 

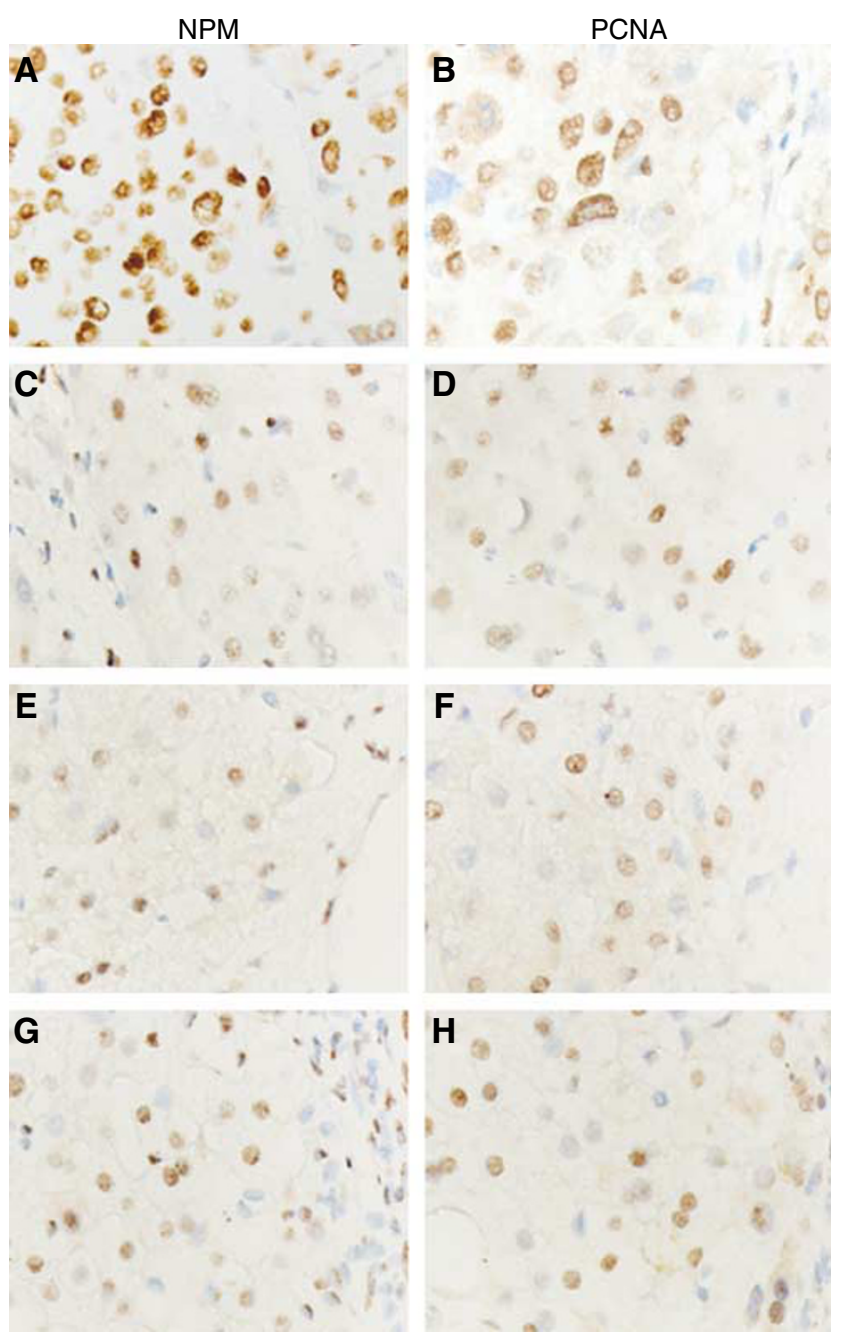

Figure 4 Nucleophosmin expression was observed in HCC and nonmalignant diseases by immunohistochemistry and compared to PCNA expression. Nuclear expression of both NPM and PCNA was stronger in the HCC tumour cells than in hepatocytes with non-malignant diseases. Images $\mathrm{A}$ and B: immunoreactivity of $\mathrm{NPM}(\mathbf{A})$ and PCNA (B) in the tumour cells with HCC; images $C$ and D: immunoreactivity of NPM (C) and PCNA (D) in the hepatocytes adjacent to the tumours with HCC; images $\mathrm{E}$ and F: Immunoreactivity of NPM (E) and PCNA (F) in hepatocytes with nodular hepatic hyperplasia; images $\mathrm{G}$ and $\mathrm{H}$ : immunoreactivity of NPM $(\mathbf{G})$ and PCNA $(\mathbf{H})$ in hepatocytes adjacent to tumours with haemangioma. All sections were counterstained with haematoxylin. (All fields $\times 400$.)

A

Comparison of NPM nuclear staining intensities among the four groups

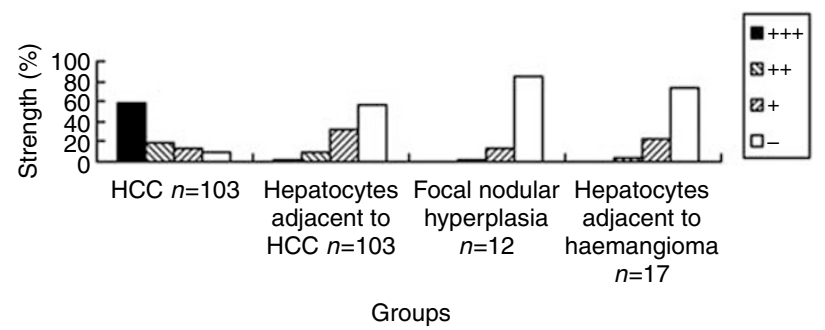

B

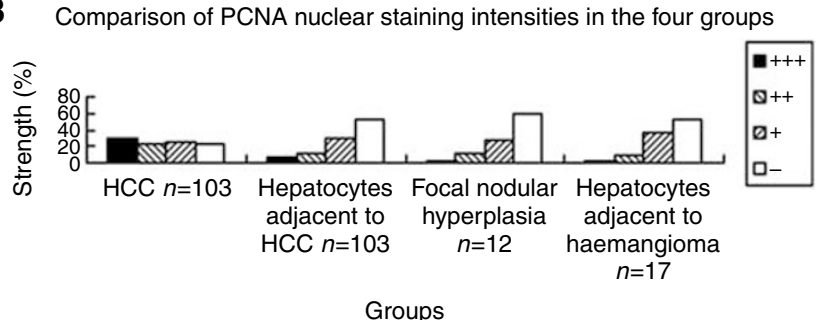

Figure 5 The staining intensities of NPM and PCNA were measured and calculated among the four groups including HCC and non-malignant liver diseases. (A) The median staining intensity percentages of NPM nuclear expression in the four groups are shown. (B) The median percentages of PCNA staining intensity in the four groups are shown.

Counting the positive cells, scored for the four staining intensities, the median percentages of NPM expression in these groups are listed in Figure 5A.

The PCNA expression levels in the four groups were also determined and analysed by immunohistochemistry (Figures 4 and 5). It was found that PCNA labelling in the cancer cells with HCC was stronger than in the hepatocytes with non-malignant diseases. The median percentages of PCNA expression divided into the four groups are listed in Figure 5B.

To statistically compare NPM or PCNA expression among the four groups, staining intensity group percentages were transformed to Ridit values representative of NPM or PCNA expression in each case. The mean Ridit values of NPM or PCNA expressions in the four groups are listed in Table 1. There were significant statistical differences of NPM and PCNA expressions among the four groups by ANOVA analysis $(P=0.000)$. Using the Student - Newman-Keuls analysis, NPM or PCNA expressions in HCC were significantly higher than in non-malignant diseases $(P<0.05)$

We also calculated the correlation of the mean Ridit values of NPM and PCNA expression by Pearson correlation analysis (Figure 6). There was statistical correlation between NPM and PCNA expression in the four groups $\left(R^{2}=0.5639\right)$.

Table I Comparison of NPM and PCNA expressions among the four groups

\begin{tabular}{|c|c|c|c|c|c|c|c|}
\hline & Cases & \multicolumn{3}{|c|}{ NPM expression } & \multicolumn{3}{|c|}{ PCNA expression } \\
\hline Group I & 103 & $0.295 \pm 0.115$ & & & $0.429 \pm 0.107$ & & \\
\hline Group 2 & 103 & $0.679 \pm 0.087$ & & & $0.624 \pm 0.084$ & & \\
\hline Group 3 & 12 & $0.603 \pm 0.097$ & & & $0.608 \pm 0.085$ & & \\
\hline
\end{tabular}

NPM = nucleophosmin; PCNA = proliferative cell nuclear antigen; ANOVA = analysis of variance. Group I: HCC; group 2: hepatocytes adjacent to the tumours with HCC; group 3: hepatocytes in the lesions with focal nodular hyperplasia; group 4: hepatocytes adjacent to the tumours with haemangioma. *Statistically significant $(P<0.0 \mathrm{I})$. 
Statistic analysis on NPM expression differences in HCC with clinical parameters

We further investigated the differences of NPM or PCNA expression in 103 cases of HCC based on different clinical parameters, including the patient's sex, age, clinical TNM stage, tumour size, tumour amount, serum level of alpha fetal protein (AFP), histological grade, and the presence or absence of liver cirrhosis. These cases were divided into two groups for each clinical parameter (Marrero et al, 2005). The mean Ridit values of each group for each clinical parameter were measured by Ridit

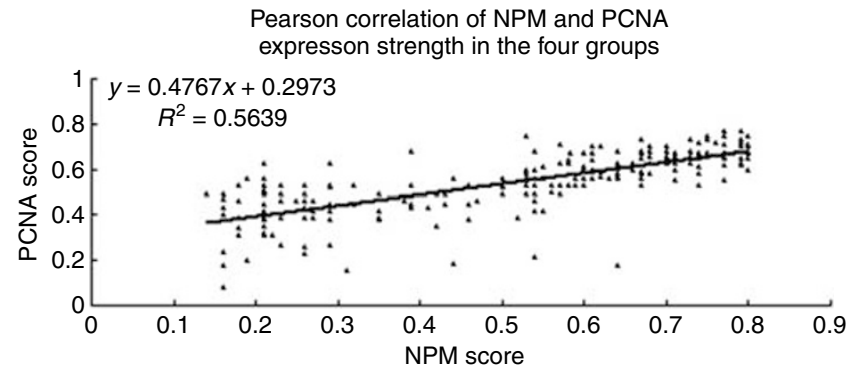

Figure 6 Potential correlations with NPM and PCNA expression were statistically analysed among the four groups including HCC and nonmalignant liver diseases. Pearson correlation of NPM and PCNA expression revealed a correlation of expression among the four groups $\left(R^{2}=0.5639\right)$. analysis and are shown in Table 2. The differences in the Ridit values for the two groups of each clinical parameter were analysed by $t$-test analysis. Significant statistical differences were found for three of the clinical parameters, including serum level of AFP, histological grade, and presence of liver cirrhosis $(P<0.05)$. These data indicate that the levels of NPM expression in HCC correlate with the grading of the tumour, and thus upregulation of NPM may be involved in tumour progression. There were no statistical differences with respect to NPM expression in the HCC cases with the other clinical parameters including patient's sex, age, clinical TNM stage, tumour size, and tumour amount $(P>0.05)$.

The differences of PCNA expression in the HCC cases based on the same clinical parameters were analysed and are shown in Table 2. We found no statistical differences in PCNA expression in the HCC cases with these clinical parameters $(P>0.05)$.

\section{Nucleophosmin expression in human normal adult and embryo tissues}

To evaluate the potential role of NPM in normal human cells, we investigated NPM expression in normal human adult and embryo tissues ( 5 months) by tissue array and immunohistochemistry. As shown in Figure 7, NPM expressions in different tissues were not quite consistent either in staining intensity or in location. Nucleophosmin was expressed in some cells from adults that did not express this protein in embryos. There were three types of NPM subcellular labelling: one was predominantly located in

Table 2 NPM and/or PCNA expressions in HCC with clinical parameters

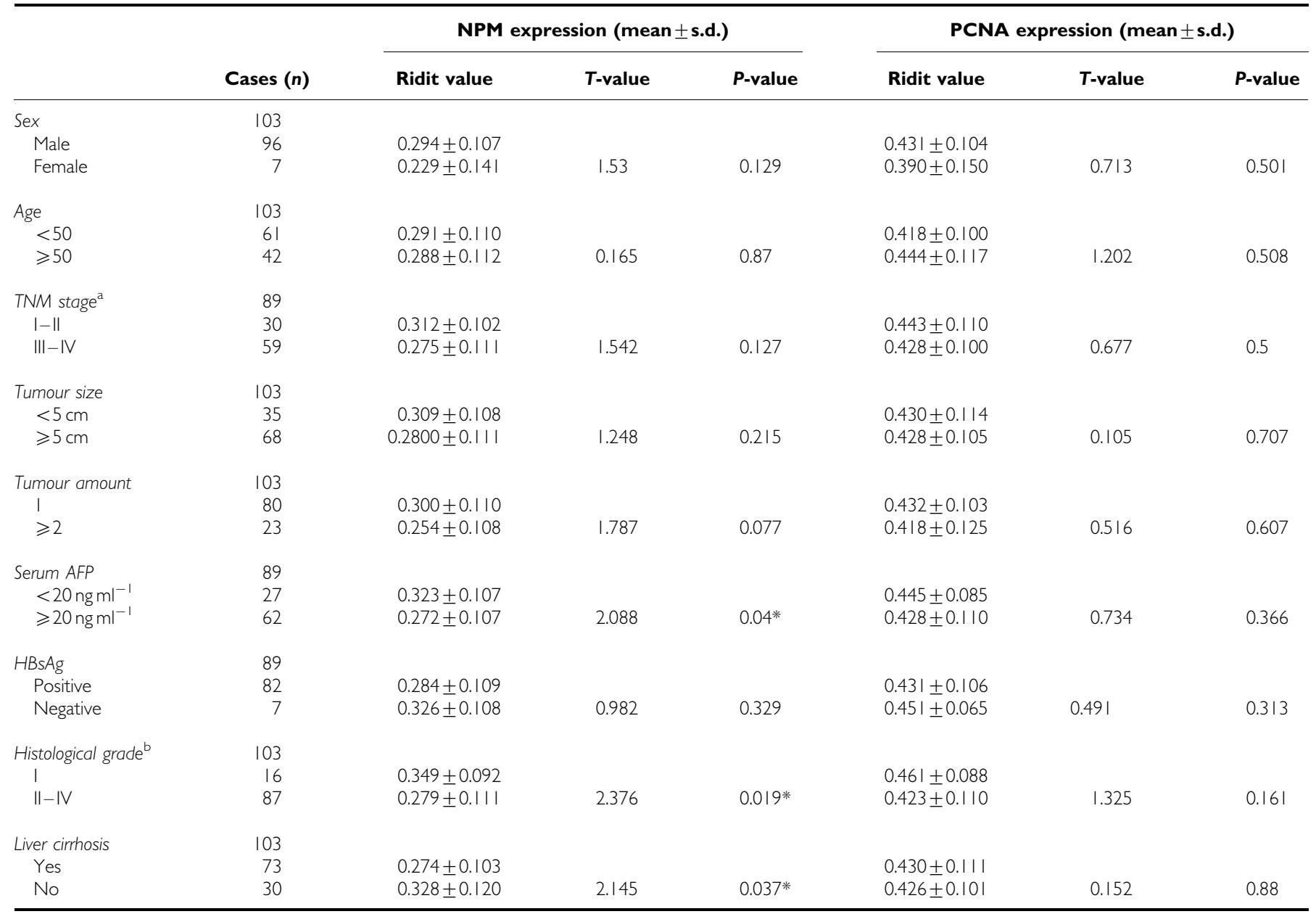

NPM = nucleophosmin; PCNA = proliferative cell nuclear antigen; $\mathrm{HCC}=$ hepatocellular carcinoma; AFP = alpha fetal protein. ${ }^{\mathrm{a}} \mathrm{TNM}$ stage was based on the UICC criteria published in 1997. ${ }^{b}$ Histological grade was with reference to WHO classification published in 2000. *Statistically significant $(P<0.05)$. 

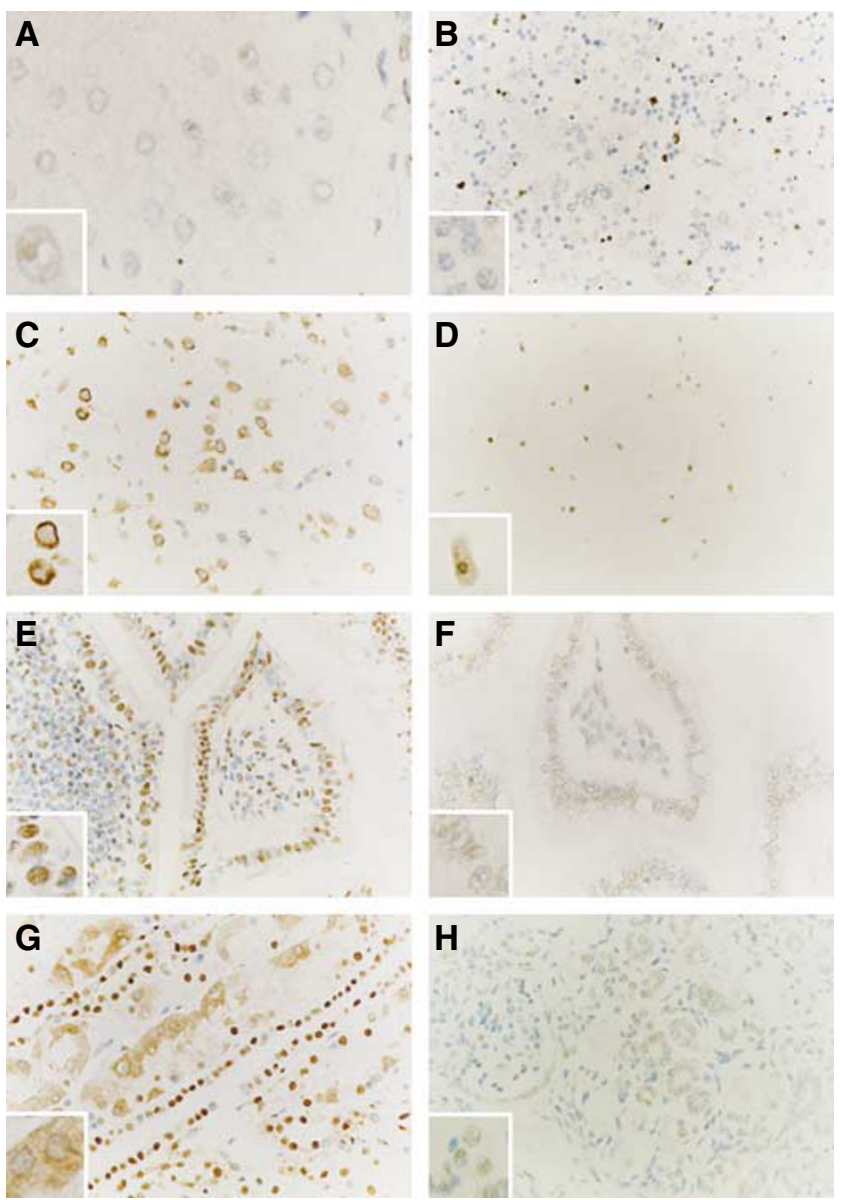

Figure 7 Nucleophosmin expression was detected in human adult and embryo tissues from different organs by immunohistochemistry. (A) Adult liver, (B) embryo liver, (C) adult brain, (D) embryo brain, (E) adult small intestine, $(\mathbf{F})$ embryo small intestine, $(\mathbf{G})$ adult kidney, and $(\mathbf{H})$ embryo kidney. We observed different subcellular localisation of NPM in human cells, such as in the nucleoli of hepatocytes in normal adult liver $(\mathbf{A})$, the nuclei of epithelia and glands $(\mathbf{D}-\mathbf{F}$ and $\mathbf{H})$, and the cytoplasm of adult cerebral neuronal cells $(\mathbf{C})$ and epithelial cells of the kidney proximal tubule (G). There was very weak labelling in embryo liver hepatocytes (B). A greater magnification of the stained cells selected from the corresponding image is shown as inset. All sections are counterstained with haematoxylin. (All fields, $\times 400$; inset images, $\times 1000$.)

nucleoli, such as in normal adult liver (Figure 7A); another was mainly in the nuclei, such as in the epidermis, epithelia, glands, and proliferating lymphocytes located in the germinal centres (Figure 7D-H); the third type showed mainly cytoplasmic staining, such as in mitotic and some non-mitotic cells, such as adult cerebral neuronal cells (Figure 7C) and the epithelial cells of the kidney's proximal tubule (Figure 7G), embryo osteoblasts, and thymic cells. Interestingly, there was only very weak labelling of NPM in the hepatocytes from normal embryo livers (Figure 7B) and expression was found in the nucleolus and nucleus of the embryo brain cells (Figure 7D). Differential expression of NPM in normal human cells demonstrated that it may correlate with cell proliferation and differentiation.

\section{Nucleophosmin expression in cancer tissue}

To evaluate the potential role of NPM in the human malignancies, we further investigated NPM expression in different cancer tissues by immunohistochemistry on tissue arrays. Strong nuclear expression of NPM was found in carcinomas originating from stratified epithelia derived from several organs, including the skin,
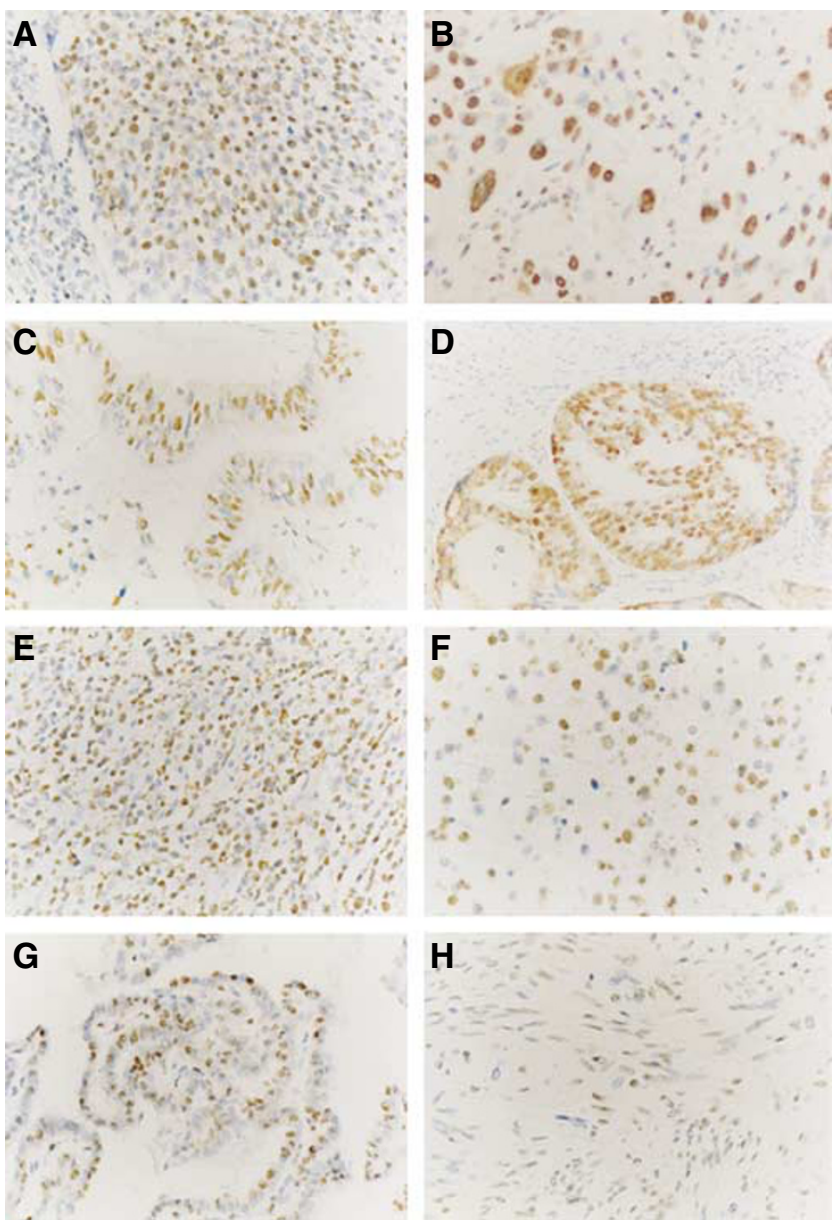

Figure 8 Nucleophosmin expression was observed in different types of human malignancies by immunohistochemistry. (A) Poorly differentiated squamous cervical carcinoma, (B) pulmonary squamous carcinoma, (C) adenocarcinoma of the small intestine, (D) adenocarcinoma of the prostate, (E) B-cell lymphoma of the testis, (F) astrocytoma of the brain (G) thyroid papillary carcinoma, and (H) Wilms' tumour of the kidney. Strongly stained NPM nuclear expression was observed in the tumour cells of many malignancies $(\mathbf{A}-\mathbf{G})$, whereas only weakly stained nuclear expression was found in the Wilms' tumour sample $(\mathbf{H})$. All sections are counterstained with haematoxylin. (All fields $\times 400$.)

oesophagus, cervix, and lung, in carcinomas from glandular epithelia from the breast, thyroid gland, lung, digestive tract, ovary, and prostate, and in other malignancies such as B-cell lymphoma, renal cell carcinoma, and glioblastoma. However, we observed only nucleolar expression and weak nuclear expression in Wilms' tumour of kidney. The NPM expressions in eight different malignancies originated from eight different organs are shown in Figure 8. In general, stronger nuclear staining of NPM expressions in the malignancies was observed as compared to those in the corresponding normal tissues (Figure 7; data not shown). Put together, NPM expression detected in human malignancies implicated a potentially important role for NPM in tumorigenesis and development.

\section{DISCUSSION}

The present study revealed four principal findings: (1) that NPM is differentially expressed, either in quantity or subcellular location, in malignant human liver cancer (HCC) compared to hepatocytes with non-malignant liver diseases. Similar differences in PCNA expression were detected among these cases. These results indicate that 
NPM might play an important role in the genesis and development of HCC. We also found that (2) in the cases with HCC, NPM overexpression was correlated with clinical parameters, such as serum AFP level, tumour pathological grading, and cirrhosis, suggesting that NPM might serve as a potential marker for HCC. (3) Nucleophosmin was differently expressed either in quantity or in subcellular location in normal human liver and other tissues when adult and embryo tissues were compared. The NPM expression of normal human cells was shown to correlate with cell proliferation and differentiation. Lastly, (4) NPM was found to be highly expressed in multiple cancers from different organs derived from either epithelial or non-epithelial origins. This NPM expression in multiple human malignancies implicates that NPM might play an important role in tumorigenesis and development.

The present study examined the potential role of NPM in HCC. Our data revealed a high level of NPM expression in the majority of HCC examined. Overexpression of NPM in HCC may thus represent an acquired phenotypic feature. Furthermore, there was a positive correlation between NPM expression and tumour pathological grading or HCC with liver cirrhosis. Therefore, NPM may be an important factor in the development of HCC. Previous studies showed that NPM seemed to be associated with both oncogenic activity and tumour suppression. Hence, overexpression of NPM in NIH3T3 cells resulted in malignant transformation (Kondo et al, 1997). Nucleophosmin nuclear overexpression in colon adenoma/ carcinoma seems also to be associated with adenoma/carcinoma progression (Nozawa et al, 1996). It has been shown that NPM overexpression was present in several human solid tumours, including gastric cancer (Tanaka et al, 1992), colon cancer (Nozawa et al, 1996), bladder cancer (Tsui et al, 2004), prostate cancer (Subong et al, 1999), thyroid cancer (Onda et al, 2004), and ovary cancer (Zhang, 2004). Interestingly, a recent review reported that NPM overexpression in tumour cells led to increased proliferation and inhibition of apoptosis and was involved in human tumorigenesis (Grisendi et al, 2006).

The present study also demonstrated that there were similarities of NPM nuclear expression and PCNA expression between HCC and non-malignant diseases. We recently observed that NPM expression in rat hepatocytes increased quantitatively and changed in subcellular location during liver regeneration after partial hepatectomy. Moreover, these alterations of hepatocyte NPM level were consistent with different phases of the cell cycle and the level of PCNA (Yun et al, 2002, 2003). Yung's group has recently shown that the expression of NPM correlates with PCNA expression. They demonstrated that the increased NPM resulted in enhanced DNA repair ability and rescued cells from UV killing (Wu et al, 2002). They further provided evidence that ectopic overexpression of NPM caused a marked upregulation in the amounts of PCNA and YY1 (a multifunctional transcriptional factor) proteins. Nucleophosmin regulates the PCNA promoter through YY1 (Weng and Yung, 2005).

Another interesting finding in this study is that there are different subcellular locations of NPM in the normal cells of the embryo and the adult. We describe three types of NPM cellular distribution found in normal human adult or embryo tissues - the nucleoli, nuclei, or the cytoplasm. Differential subcellular expression of NPM in normal human cells may correlate with cell proliferation and differentiation. Previous reports have shown that NPM is involved in the assembly and/or intranuclear transport of pre-ribosomal particles and in cytoplasmic/nuclear trafficking (Spector et al, 1984; Yung et al, 1985; Borer et al, 1989). In mitotic cells, most nuclear proteins were dispersed throughout the cytoplasm, including NPM, which then re-localised to the centrosomes, and later to each daughter cell that received one centrosome bound by NPM after mitosis (Okuda et al, 2000). Interestingly, we also found that some of the non-mitotic cells, such as adult cerebral neuronal cells, epithelial cells of the adult kidney proximal tubule, and embryo osteoblast and thymic cells contain cytoplasmic NPM. The functional significance and the mechanism behind this cytoplasmic localisation remain to be determined. Previous studies found that two isoforms of NPM, termed B23.1 and B23.2, were expressed in different subcellular locations of human cells and possessed different functions (Chang and Olson, 1990; Umekawa et al, 2001). B23.1 was primarily localised in nucleoli, where it was likely targeted to rRNA-rich nucleoli, possibly owing to its RNAbinding activity, whereas B23.2 was localised in the nucleoli and also throughout the nucleoplasm; the function of B23.2 is not yet well understood. These two proteins are identical except for the Cterminal 35 amino acids observed in B23.1 that is absent in B23.2. The expression level of B23.2 was much lower than that of B23.1. Interestingly, the expression level of B23.1 was closely correlated with the cell growth rate, whereas that of B23.2 remained relatively constant (Wang et al, 1993).

Another previous study demonstrated the cytoplasmic location of NPM and the mutation of exon 12 of the NPM1 gene as a hallmark of a large subgroup of primary acute myelogenous leukaemia (NPMc + AML) (Falini et al, 2005). However, we have not observed cytoplasmic NPM in any of the 103 cases of HCC or other cancers in the tissue array used in the present study. The cytoplasmic location in the solid cancers will be worthy of further study.

Both polyclonal and monoclonal antibodies to NPM have been reported in the past, but only a few immunocytochemical analyses or distribution studies of NPM in human tissues have confirmed its ubiquitous expression in both normal and neoplastic cells (Nozawa et al, 1996; Cordell et al, 1999; Subong et al, 1999). The study describes monoclonal antibodies that detect NPM in paraffin-embedded tissue array samples and confirms NPM distribution within different cells.

Altogether, the present study provides evidence that NPM may be involved in hepatocarcinogenesis and serve as a potential marker for applied clinicopathology in HCC.

\section{ACKNOWLEDGEMENTS}

We thank ProMab Biotechnologies Inc. (Changsha city, China) for providing us technical support on the production of recombinant NPM protein and development of the monoclonal antibodies against human NPM. We thank Mr Jinhui Hou and Mrs Meiqing Zhao for their excellent technical assistance with immunohistochemistry, Professor Li Lin and Mr Jun Liu for their kind assistance in statistics, and Mr Zhiyi Zhang, Ms Shuguang Su, and Ms Meifang Zhang for their help in sample collection and preparation. We also express our gratitude to Dr Yanping Zhang, Lineberger Comprehensive Cancer Center and Department of Radiation Oncology, The University of North Carolina at Chapel Hill, NC, USA for editing the manuscript and critical comments on the study. This was supported by grants from National Natural Science Foundation of China (no. 30471960) and Guangzhou City Scientific and Technological Bureau (no. 2002Z3-j20091).

\section{REFERENCES}

Bertwistle D, Sugimoto M, Sherr CJ (2004) Physical and functional interactions of the Arf tumor suppressor protein with nucleophosmin/ B23. Mol Cell Biol 24: $985-996$

Borer RA, Lehner CF, Eppenberger HM, Nigg EA (1989) Major nucleolar proteins shuttle between nucleus and cytoplasm. Cell 56: 379-390
Brady SN, Yu Y, Maggi Jr LB, Weber JD (2004) ARF impedes NPM/B23 shuttling in an Mdm2-sensitive tumor suppressor pathway. Mol Cell Biol 24: $9327-9338$

Chang JH, Olson MO (1990) Structure of the gene for rat nucleolar protein B23. J Biol Chem 265: 18227-18233 
Colombo E, Marine JC, Danovi D, Falini B, Pelicci PG (2002) Nucleophosmin regulates the stability and transcriptional activity of p53. Nat Cell Biol 4: 529-533

Cordell JL, Pulford KA, Bigerna B, Roncador G, Banham A, Colombo E, Pelicci PG, Mason DY, Falini B (1999) Detection of normal and chimeric nucleophosmin in human cells. Blood 93: 632-642

Deane NG, Parker MA, Aramandla R, Diehl L, Lee WJ, Washington MK, Nanney LB, Shyr Y, Beauchamp RD (2001) Hepatocellular carcinoma results from chronic cyclin D1 overexpression in transgenic mice. Cancer Res 61: $5389-5395$

Falini B, Mecucci C, Tiacci E, Alcalay M, Rosati R, Pasqualucci L, La Starza R, Diverio D, Colombo E, Santucci A, Bigerna B, Pacini R, Pucciarini A, Liso A, Vignetti M, Fazi P, Meani N, Pettirossi V, Saglio G, Mandelli F, Lo-Coco F, Pelicci PG, Martelli MF, GIMEMA Acute Leukemia Working Party (2005) Cytoplasmic nucleophosmin in acute myelogenous leukemia with a normal karyotype. $N$ Engl J Med 352: 254-266

Feuerstein N, Mond JJ (1987) 'Numatrin', a nuclear matrix protein associated with induction of proliferation in B lymphocytes. J Biol Chem 262: $11389-11397$

Feuerstein N, Spiegel S, Mond JJ (1988) The nuclear matrix protein, numatrin (B23), is associated with growth factor-induced mitogenesis in Swiss 3T3 fibroblasts and with T lymphocyte proliferation stimulated by lectins and anti-T cell antigen receptor antibody. J Cell Biol 107: 16291642

Gao H, Jin S, Song Y, Fu M, Wang M, Liu Z, Wu M, Zhan Q (2005) B23 regulates GADD45a nuclear translocation and contributes to GADD45ainduced cell cycle G2-M arrest. J Biol Chem 280: 10988-10996

Gordon S, Akopyan G, Garban H, Bonavida B (2006) Transcription factor YY1: structure, function, and therapeutic implications in cancer biology. Oncogene 25: 1125-1142

Grisendi S, Bernardi R, Rossi M, Cheng K, Khandker L, Manova K, Pandolfi PP (2005) Role of nucleophosmin in embryonic development and tumorigenesis. Nature 437: $147-153$

Grisendi S, Mecucci C, Falini B, Pandolfi PP (2006) Nucleophosmin and cancer. Nat Rev Cancer 6: 493-505

Hingorani K, Szebeni A, Olson MO (2000) Mapping the functional domains of nucleolar protein B23. J Biol Chem 275: 24451-24457

Huang N, Negi S, Szebeni A, Olson MO (2005) Protein NPM3 interacts with the multifunctional nucleolar protein B23/nucleophosmin and inhibits ribosome biogenesis. J Biol Chem 280: 5496-5502

Itahana K, Bhat KP, Jin A, Itahana Y, Hawke D, Kobayashi R, Zhang Y (2003) Tumor suppressor ARF degrades B23, a nucleolar protein involved in ribosome biogenesis and cell proliferation. Mol Cell 12: $1151-1164$

Kondo T, Minamino N, Nagamura-Inoue T, Matsumoto M, Taniguchi T, Tanaka N (1997) Identification and characterization of nucleophosmin/ B23/numatrin which binds the anti-oncogenic transcription factor IRF-1 and manifests oncogenic activity. Oncogene 15: 1275-1281

Li J, Zhang X, Sejas DP, Bagby GC, Pang Q (2004) Hypoxia-induced nucleophosmin protects cell death through inhibition of p53. J Biol Chem 279: 41275 - 41279

Maiguel DA, Jones L, Chakravarty D, Yang C, Carrier F (2004) Nucleophosmin sets a threshold for p53 response to UV radiation. $\mathrm{Mo}$ Cell Biol 24: $3703-3711$

Marrero JA, Fontana RJ, Barrat A, Askari F, Conjeevaram HS, Su GL, Lok AS (2005) Prognosis of hepatocellular carcinoma: comparison of 7 staging systems in an American cohort. Hepatology 41: 707-716

Miao J, Chen GG, Chun SY, Lai PP (2006) Hepatitis B virus X protein induces apoptosis in hepatoma cells through inhibiting $\mathrm{Bcl}-\mathrm{xL}$ expression. Cancer Lett 236: $115-124$

Nozawa Y, Van Belzen N, Van der Made AC, Dinjens WN, Bosman FT (1996) Expression of nucleophosmin/B23 in normal and neoplastic colorectal mucosa. J Pathol 178: $48-52$

Okuda M (2002) The role of nucleophosmin in centrosome duplication. Oncogene 21: $6170-6174$

Okuda M, Horn HF, Tarapore P, Tokuyama Y, Smulian AG, Chan PK, Knudsen ES, Hofmann IA, Snyder JD, Bove KE, Fukasawa K (2000) Nucleophosmin/B23 is a target of CDK2/cyclin E in centrosome duplication. Cell 103: $127-140$
Onda M, Emi M, Yoshida A, Miyamoto S, Akaishi J, Asaka S, Mizutani K, Shimizu K, Nagahama M, Ito K, Tanaka T, Tsunoda T (2004) Comprehensive gene expression profiling of anaplastic thyroid cancers with cDNA microarray of 25344 genes. Endocr Relat Cancer 11: $843-854$

Pulford K, Morris SW, Mason DY (2001) Anaplastic lymphoma kinase proteins and malignancy. Curr Opin Hematol 8: 231-236

Schnittger S, Schoch C, Kern W, Mecucci C, Tschulik C, Martelli MF, Haferlach T, Hiddemann W, Falini B (2005) Nucleophosmin gene mutations are predictors of favorable prognosis in acute myelogenous leukemia with a normal karyotype. Blood 106: 3733 - 3739

Sicklick JK, Li YX, Jayaraman A, Kannangai R, Qi Y, Vivekanandan P, Ludlow JW, Owzar K, Chen W, Torbenson MS, Diehl AM (2006) Dysregulation of the Hedgehog pathway in human hepatocarcinogenesis. Carcinogenesis 27: 748-757

Spector DL, Ochs RL, Busch H (1984) Silver staining, immunofluorescence, and immunoelectron microscopic localization of nucleolar phosphoproteins B23 and C23. Chromosoma 90: 139-148

Subong EN, Shue MJ, Epstein JI, Briggman JV, Chan PK, Partin AW (1999) Monoclonal antibody to prostate cancer nuclear matrix protein (PRO: 4-216) recognizes nucleophosmin/B23. Prostate 39: 298-304

Szebeni A, Hingorani K, Negi S, Olson MO (2003) Role of protein kinase CK2 phosphorylation in the molecular chaperone activity of nucleolar protein b23. J Biol Chem 278: $9107-9115$

Takemura M, Ohta N, Furuichi Y, Takahashi T, Yoshida S, Olson MO, Umekawa H (1994) Stimulation of calf thymus DNA polymerase alpha activity by nucleolar protein B23. Biochem Biophys Res Commun 199: $46-51$

Takemura M, Sato K, Nishio M, Akiyama T, Umekawa H, Yoshida S (1999) Nucleolar protein B23.1 binds to retinoblastoma protein and synergistically stimulates DNA polymerase alpha activity. J Biochem (Tokyo) 125: 904-909

Tanaka M, Sasaki H, Kino I, Sugimura T, Terada M (1992) Genes preferentially expressed in embryo stomach are predominantly expressed in gastric cancer. Cancer Res 52: 3372-3377

Tsui KH, Cheng AJ, Chang PL, Pan TL, Yung BY (2004) Association of nucleophosmin/B23 mRNA expression with clinical outcome in patients with bladder carcinoma. Urolog 64: 839-844

Umekawa H, Sato K, Takemura M, Watanabe Y, Usui S, Takahashi T, Yoshida S, Olson MO, Furuichi Y (2001) The carboxyl terminal sequence of nucleolar protein B23.1 is important in its DNA polymerase alphastimulatory activity. J Biochem (Tokyo) 130: 199-205

Verheggen C, Almouzni G, Hernandez-Verdun D (2000) The ribosomal RNA processing machinery is recruited to the nucleolar domain before RNA polymerase I during Xenopus laevis development. J Cell Biol 149: $293-306$

Wang D, Umekawa H, Olson MO (1993) Expression and subcellular locations of two forms of nucleolar protein B23 in rat tissues and cells. Cell Mol Biol Res 39: 33-42

Weng JJ, Yung BY (2005) Nucleophosmin/B23 regulates PCNA promoter through YY1. Biochem Biophys Res Commun 335: 826-831

Wu MH, Chang JH, Yung BY (2002) Resistance to UV-induced cell-killing in nucleophosmin/B23 over-expressed NIH 3T3 fibroblasts. Enhancement of DNA repair and up-regulation of PCNA in association with nucleophosmin/B23 over-expression. Carcinogenesis 23: $93-100$

Yun J, Liew CT, Chew EC, Chan JY (2002) [Expression and relocation of $\mathrm{B} 23$ in the process of rat liver cell hyperplasia and liver regeneration]. Zhonghua Yi Xue Za Zhi (Chinese) 82: 974-978

Yun JP, Chew EC, Liew CT, Chan JY, Jin ML, Ding MX, Fai YH, Li HK, Liang XM, Wu QL (2003) Nucleophosmin/B23 is a proliferate shuttle protein associated with nuclear matrix. J Cell Biochem 90: 1140-1148

Yung BY, Busch H, Chan PK (1985) Translocation of nucleolar phosphoprotein B23 $(37 \mathrm{kDa} / \mathrm{pI} 5.1)$ induced by selective inhibitors of ribosome synthesis. Biochim Biophys Acta 826: 167-173

Zeller KI, Haggerty TJ, Barrett JF, Guo Q, Wonsey DR, Dang CV (2001) Characterization of nucleophosmin (B23) as a Myc target by scanning chromatin immunoprecipitation. J Biol Chem 276: 48285-48291

Zhang Y (2004) The ARF-B23 connection: implications for growth control and cancer treatment. Cell Cycle 3: 259-262 